\title{
A case of schizencephaly
}

\author{
Ozan Turamanlar ${ }^{1}$, Oğuz Kırpıko², Alpay Haktanır ${ }^{2}$, Oğuz Aslan Özen ${ }^{3}$ \\ ${ }^{1}$ Department of Anatomy, School of Medicine, Kocatepe University, Afyonkarabisar, Turkey \\ ${ }^{2}$ Department of Radiology, School of Medicine, Kocatepe University, Afyonkarahisar, Turkey \\ ${ }^{3}$ Department of Anatomy, School of Medicine, Namık Kemal University, Tekirdă̆, Turkey
}

\begin{abstract}
Schizencephaly is a rare congenital disorder of cerebral cortical development. It is a neuronal migration anomaly, caused by insults to migrating neuroblasts during 3rd to 5th gestational months. We encountered schizencephaly in the cranial magnetic resonance imaging (MRI) of a 4 month-old male baby. MRI demonstrated wide clefts occupying parietal regions bilaterally and the right occipital region partly. These areas were connected with lateral ventricles and also filled with cerebrospinal fluid. Although prevalence of this disorder is quite low and its incidence is unknown and also there may be no clear symptoms as in our case, we emphasize that it should not be overlooked in differential diagnosis.
\end{abstract}

Key words: cranial; MRI; neurodevelopment anomaly; schizencephaly

Anatomy 2012-2013;6-7:65-67, (c) 2012-2013 TSACA

\section{Introduction}

Schizencephaly is a rare congenital disorder of cerebral cortical development. It is a neuronal migration anomaly, caused by insults to migrating neuroblasts during 3 rd to 5 th gestational months. ${ }^{[1]}$ Other anomalies in this category include agyria, pachygyria, polimicrogyria, unilateral megalencephaly, gray matter heterotopias, lissencephaly and agenesis of corpus callosum. ${ }^{[2,3]}$ Basically, schizencephaly is characterized by a cleft traversing cerebral hemisphere, infolding of cortical gray matter along the cleft, an abnormal ventricular system and other associated cerebral anomalies. ${ }^{[4]}$ Congenital cleft of the cerebral mantle extends from the pial surface to the lateral ventricle. Schizencephalic cleft occurs more often in the anterior than in posterior neocortex. It is commonly observed in the posterior frontal and parietal regions of the anterior neocortex. ${ }^{[5]}$ Clefts most commonly involve the parasylvian region and have been divided in two subtypes. Closed-lip schizencephaly (Type 1), with narrow clefts and lips fused in certain areas (pial-ependymal seam); and open-lip schizencephaly (Type 2) with separated walls encompassing an excessive cerebrospinal fluid space. ${ }^{[6]}$
There are four categories defined radiologically i.e. unilateral open lip (36.2\%), unilateral closed lip (25.6\%), bilateral open lip (25.6\%) and bilateral closed lip (12.8\%). Closed lip schizencephaly usually presents with hemiparesis or motor delay, whereas patients with open lip schizencephaly is present with hydrocephalus. ${ }^{[7]}$

\section{Case Report}

We encountered schizencephaly in the cranial MRI of a 4 month-old male baby. There was no definite clinical history except weakness of suckling. Both of the parietal lobes were not seen in the MRI (Figures 1 and 2). MRI also demonstrated areas which occupied parietal regions bilaterally and right occipital region partly (Figure 3). These areas were connected with lateral ventricles and also filled with cerebrospinal fluid (CSF). This appearance was consistent with schizencephaly type II (open lips).

MRI imaging was performed under general anesthesia. However, considering the age and condition of the patient, unwanted movements of the patient caused artifacts in MR images. 


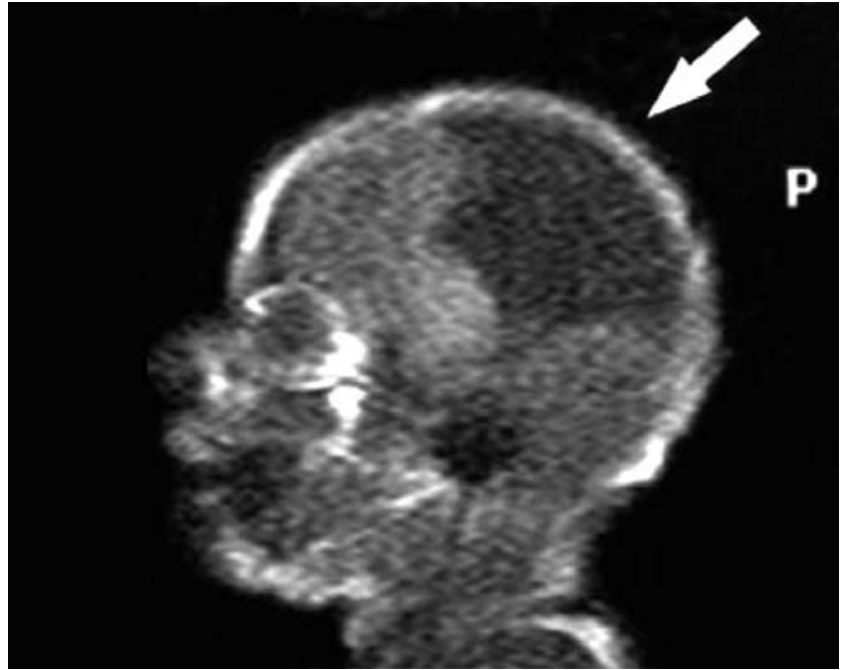

Figure 1. A wide CSF cleft is seen occupying the parietal lobe (arrow) in the T1 weighted sagittal MRI image.

\section{Discussion}

Schizencephaly may occur due to various causes. It may be due to localized ischemia in periventricular germinal matrix, or it may be an extreme variant of cortical dysplasia, in which the infolding of cortex extends all the way into the lateral ventricle. A report of schizencephally in two affected sibs suggested genetic basis and indicated the possibility of autosomal recessive form of transmission. Schizencephaly has recently been associated with a germline mutation in homeobox gene EMX2 in $70 \%$ of patients. CMV infection, postnatally, has also been blamed in its complex multifactorial pathogenesis. Intoxicants, radiation and intrauterine exposure to warfarin have also been associated with this anomaly. ${ }^{[1]}$

The schizencephalic cleft in our case was extending to lateral ventricle. This is consistent with the definition of schizencephaly, however, there are number of case reports of schizencephaly involving the third ventricle.

Age at detection ranges from 8 months to 30 years. Clinical manifestations vary from mild to severe and include developmental delay and retardation, microcephaly, language dysfunction, focal or generalized motor abnormalities and seizures. ${ }^{[4]}$ However, in our case the only distinct clinical finding was developmental delay which was manifested as weakness of suckling.

Cerebrospinal fluid pulsation from the lateral ventricles with resultant pressure effects on the inner table of the skull vault is a well-described feature of open lip anomaly ${ }^{[8]}$ This may emphasize the importance of physical examination in infants with open lip schizencephaly as in our case.

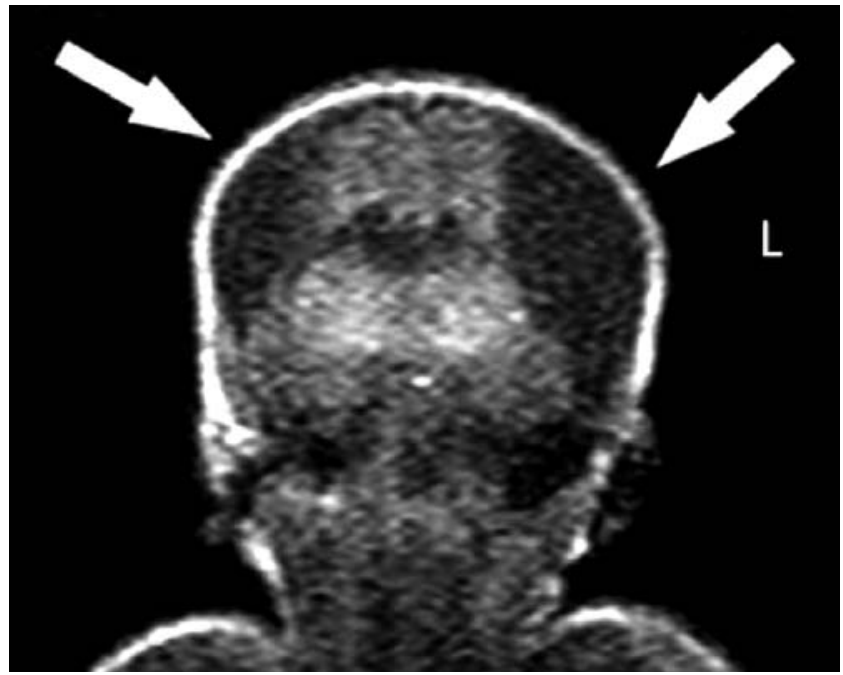

Figure 2. Bilateral parietal clefts (arrows) are demonstrated in the T1 weighted coronal MRI image.

Neurodevelopmental outcome is generally poor with $51 \%$ showing severe deficits and $32 \%$ moderate impairment. However, $17 \%$ have mild or no problem. In our case, data was not available about the neurodevelopmental outcome of the patient. Open lip schizencephaly is more likely to be associated with worse outcome than closed lip schizencephaly. Thus presentation and outcome vary but are related to extent of cortical involvement. Mental retardation is common with bilateral clefts. ${ }^{\left[{ }^{[9]}\right.}$ Although prevalence of this disorder is quite low and its incidence is unknown and also there may be no clear symptoms, as in our case, we emphasize that it should not be overlooked in differential diagnosis.

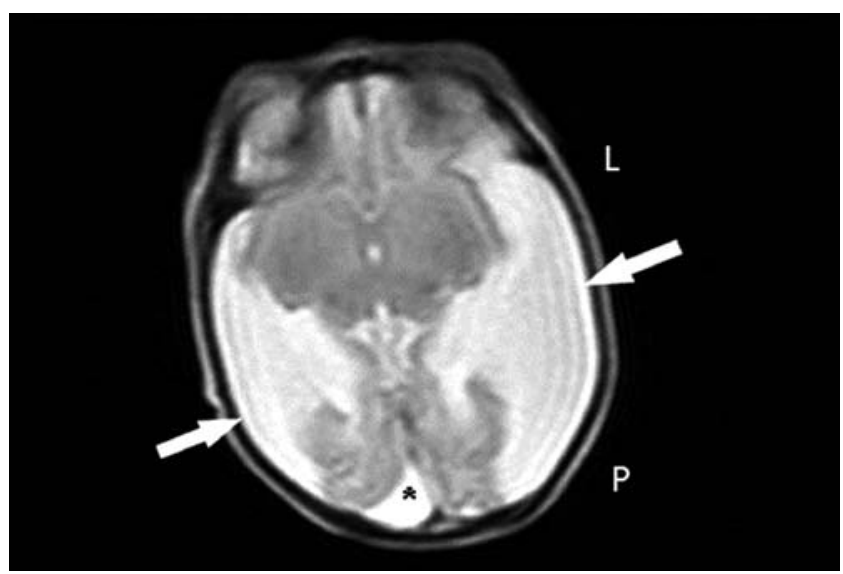

Figure 3. T2 weighted transverse MRI demonstrating wide clefts occupying parietal regions bilaterally (arrows) and right occipital region partly (asterisk). These areas are connected with lateral ventricles and also filled with CSF. 


\section{References}

1. Relan P, Chaturvedi SK, Shetty B. Schizencephaly associated with bipolar affective disorder. Neurol India 2002;50:194-7.

2. Barkovich AJ, Chuang SH, Norman D. Magnetic resonance of neuronal migration anomalies. AJR Am J Roentgenol 1988;150: 179-87.

3. Velez-Dominguez LC. Neuronal migration disorders. Gac Med Mex 1998;134:207-15.

4. Miller GM, Stears JC, Guggenheim MA, Wilkening GN. Schizencephaly: a clinical and CT study. Neurology 1984;84:9971001 .
5. Sener RN. Schizencephaly and congenital cytomegalovirus infection. J Neuroradiol 1998;25:151-2.

6. Sitnikov AR. Clinical case of the late diagnosis of type-II schizencephaly. Rural Remote Health 2007;7:661.

7. Packard AM, Miller VS, Delgado MR. Schizencephaly: correlations of clinical and radiological features. Neurology 1997;48: 1427-34.

8. Srikanth SG, Jayakumar PN, Vasudev MK. Open and minimally open lips schizencephaly. Neurol India 2000;48:155-7.

9. Denis D, Chateil JF, Brun M, et al. Schizencephaly: clinical and imaging features in 30 infantile cases. Brain Dev 2000;22:475-83.

\footnotetext{
Correspondence to: Ozan Turamanlar, MD, PhD Department of Anatomy, Faculty of Medicine, Afyon Kocatepe University Afyonkarahisar, Turkey Phone: +90 2724440303 Fax: +90 2722281417 e-mail: ozanturamanlar@hotmail.com Conflict of interest statement: No conflicts declared.
} 